\title{
Focused Modularity : Rapid Iteration of Design and Fabrication of a Meter-Scale Hexapedal Robot
}

\author{
D. Miller, I. Fitzner, S. B. Fuller, and S. Revzen* \\ EECS Dept., U. Michigan, Ann Arbor, MI 48109, USA \\ *E-mail: shrevzen@umich.edu
}

\begin{abstract}
Robots are faced with increasingly complex, hard to simulate environments. Multilegged robots exhibit dynamics that are often difficult to model well. Their development thus depends on experimental verification. Here we propose "focused modularity" - an approach combining benefits of both modular robotics and more traditional rapid fabrication. We made robot mechanisms by laser cutting low-cost foam board, while the complexity of motor control and electronics is focused in a small number of servomotor modules. This dramatically reduced the cost and time of design iteration, and allowed incremental improvements to make up for deficits in material strength and manufacturing tolerance. We present meter-scale hexapods whose chassis and drive train were manufactured for under 20 USD and under four hours of skilled labor. To date we have produced over 40 revisions of the design, including 25 iterations of drive mechanism design and 7 full robot prototypes built. This represents a substantially more thorough exploration of the design space than could be possible using conventional development approaches. We suggest a library of suitable robot designs to be developed, permitting a robot to be built on the fly with functionality for a particular task as the situation demands.
\end{abstract}

INTRODUCTION - Classical engineering designs closed loop interactions of robots with their environment using accurate validated models, and sound application of mathematically provable control methods. For legged robots in uncontrolled environments, the requisite models of the environment and robot-environment interaction are rarely reliable. At the same time, these robots are coming into broader use where cost drives down manufacturing tolerances and reliability of robot components. Our challenge is to obtain reliable legged robot behaviors from cheaper, inaccurately characterized parts.

Here we explore one approach toward this goal, which we call "focused modularity". While traditional modular robots aim to construct the entire robot from modular parts, we focus our modularity in few high-complexity 
reusable subsystems. We concentrated the electronics and actuation in a small number of general-purpose motor modules that attach to the chassis via a snap-on interface or large bolts, and daisy-chain for power and communications.

This approach differs from robotics kits $^{1}$ or classical modular robots, ${ }^{2}$ in that we assume availability of rapid manufacturing tools, rather than relying entirely on pre-fabricated parts. We use modularity to localize complexity and speed up assembly, rather than as an overarching paradigm. Using our approach we built a meter-scale hexapedal robot — far larger than commonly available 3D printing allows. This robot can walk, steer, and turn in place on surfaces indoors and outdoors in the U. Michigan Ann Arbor campus, testifying to its viability as a legged platform.

The robot chassis and drive-train of our robots borrow from centimeter scale and millimeter scale robots made with the Smart Composite Microstructures (SCM) SCM uses a laser to cut planar materials. Thest are laminated together and folded to produce 3D linkages. SCM and related methods produced many robotic prototypes, such as a $3 \mathrm{~cm}$ six-legged hexapod walker made of fiberglass-reinforced composites, ${ }^{3}$ a larger $10 \mathrm{~cm}$ hexapod built from fiberboard, ${ }^{4}$ a $1 \mathrm{~cm}$ scale hovering robot fly, ${ }^{5}$ centipede, ${ }^{6}$ and other structures such as linked chains. ${ }^{7}$ The speed of laser machining contributes to rapid design iteration. However, the maximum size of robots manufactured by SCM hitherto is approximately $10 \mathrm{~cm}$. We contribute the first human-scaled mechanisms built using these techniques. Our primary

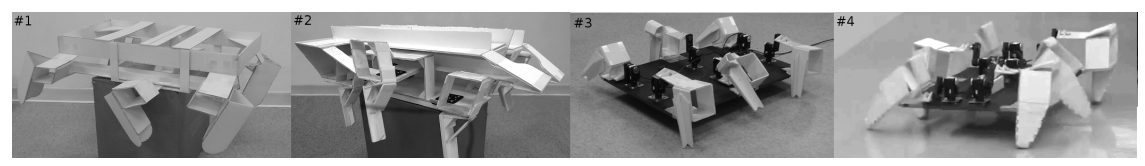

Fig. 1. Four generations of the robot chassis. \#1 explored kinematics and had no actuators; \#2 is scaled up Berkeley design; \#3 first version able to walk; \#4 improved leg and drive-train design. The structural material is foamboard.

contribution is showing how focusing modularity gives rapid iterations of mechanical design at low cost, opening the door to: (1) designing robots (legged or otherwise) based on experimentation rather than simulation; and (2) fabricating them on-the-fly when they are needed. A secondary contribution is an SCM-like process for rapid fabrication of low cost mechanisms at human scales. We primarily describe our \#4 and \#6 design iterations, which were $76 \mathrm{~cm}$ long ambulatory robotic hexapods. Each chassis, including legs and drive-train, cost less than 20 USD, and required less than four hours of skilled labor to build. Figure 2 lists design changes in terms of 
drive mechanism changes, complete chassis re-builds, and other leg and mechanism revisions. Over 40 revisions were made and tested at a cost (in time and money) normally associated with only a few iterations of robot design. Rapidly and cheaply exploring the design space and experimentally testing the results, we produced an effectively moving legged robot, whose operation was incrementally improved in the actual real world environment in which it operates.

Fig. 2. Design Revision History

\begin{tabular}{|l|c|c|c|c|c|c|c|c|}
\hline Chassis version & 1 & 2 & 3 & 4 & 5 & 6 & 7 & total 7 \\
Drive-Train revisions & 5 & 6 & 2 & 4 & 5 & 1 & 1 & total 24 \\
Leg / Other revisions & 5 & 15 & 2 & 5 & 10 & 3 & 2 & total 42 \\
Label in Fig.1 & $\# 1$ & $\# 2$ & $\# 3$ & $\# 4$ & & \\
\hline
\end{tabular}

BACKGROUND on SCM - Our goal was to create a family of robot designs that can be fabricated quickly and inexpensively from few materials with few tools. Smart Composite Microstructures $(\mathrm{SCM})^{8}$ provides a rich collection of robot mechanisms designed for the centimeter and millimeter scale.

The process consists of: (1) Cutting a rigid material in a laser cutter leaving gaps for flexure joints. (2) Laminating the compliant flexure material on that lower rigid plate. (3) Placing and cutting an upper rigid plate. (4) Curing the materials to make them adhere. (5) Releasing the mechanism and locking it in shape.

MATERIAL SELECTION - To make an SCM-like process compatible with parts at the meter scale, we first selected an appropriate plate material. Our criteria were availability, low cost, and high rigidity. Additionally, material needed to be machinable in our Universal Laser Systems model PLS6.150D, $150 \mathrm{~W} \mathrm{CO}_{2}$ laser cutter.

Candidates for the plate material were foam-core board (Elmer's Products Inc. $50.8 \mathrm{~cm} \times 76.2 \mathrm{~cm} \times 0.72 \mathrm{~cm}$ foam-core board), corrugated plastic board (SABIC Polymershapes Coroplast COR-2436 $91.4 \mathrm{~cm} \times 61.0 \mathrm{~cm} \times$ $0.40 \mathrm{~cm}$ ), and corrugated cardboard (Home Depot $55.9 \mathrm{~cm} \times 55.9 \mathrm{~cm} \times$ $53.3 \mathrm{~cm}$ box). Price per square meter for foam-core was $\$ 14.03$ (OfficeMax), Corrugated Plastic $\$ 22.21$ (DisplayShops) and corrugated cardboard $\$ 1.05$ (Home Depot) .

We quantified each material's resistance to bending via its mass-specific flexural rigidity, the ratio $E I / M=\left(P l^{3}\right) /(3 M \delta)$, which assumes an Euler cantilever beam bending model ${ }^{9}$ with load $P$ and length $l$.

We bended single layers of each material by securing $5 \times 20 \mathrm{~cm}$ samples 
to a table with a rectangular steel mass such that $15 \mathrm{~cm}$ of the sample extended off the table. We tested 3 samples of each material at 2 loads (143土 $0.1 \mathrm{~g}, 257 \pm 0.1 \mathrm{~g}$ ), taping the entire length of the end of the sample to the entire length of the test mass. We measured the resulting deflections with a ruler (error $\pm 1 \mathrm{~mm}$ ). For non-isotropic materials, we tested both strong and weak directions (ie. along the corrugations and across the corrugations).

\begin{tabular}{c|cc|cc|c} 
& \multicolumn{2}{|c|}{ Plastic } & \multicolumn{2}{c|}{ Cardboard } & Foam-Core \\
& (strong) & (weak) & (strong) & (weak) & \\
\hline$M(\mathrm{~g})$ & $7.2 \pm 0.2$ & $7.2 \pm 0.2$ & $0.24 \pm 0.02$ & $0.19 \pm 0.02$ & $6.4 \pm 0.5$ \\
\hline$\frac{E I}{M}\left(\frac{\mathrm{m}^{3}}{\mathrm{~s}^{2}}\right)$ & $30 . \pm 2$. & $10.3 \pm 0.4$ & $38.3 \pm 2.3$ & $31 . \pm 2.5$ & $110 \pm 20$
\end{tabular}

The foam-core board is superior to the other materials in terms of massspecific rigidity, and was therefore selected. It consists of a rigid foam sandwiched between sheets of fiberboard.

We selected composite fiber tape (3M, Scotch \#8959; $26 \mathrm{k} \mathrm{N} / \mathrm{m}$ strength; $6 \%$ yield strain) as our flexure material as the plastic is compliant to bending while the fibers provide support against tensile loads. Adhesive tape allowed us to skip the curing and lamination step of the SCM process.

Experience showed us that a common mode of failure is when foam-core fiberboard de-laminates from the foam filling. We protected against this by "frapping" - the wrapping of additional tape fully around the edge of the foam-core plate (see Fig. 3). For this we used a unidirectional fiber tape (3M, Scotch, \#898, $66.5 \mathrm{k} \mathrm{N} / \mathrm{m}$ strength; $5 \%$ yield strain) for frapping.

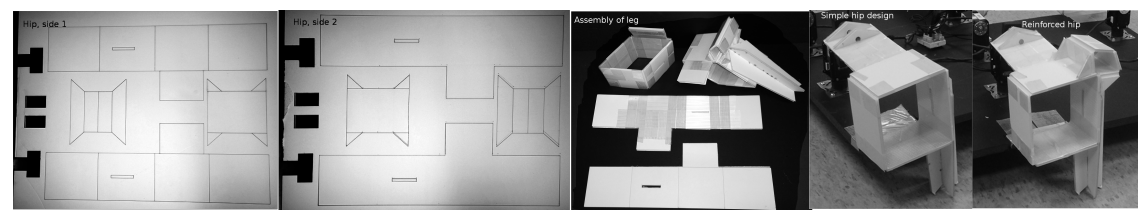

Fig. 3. Laser cuts of a hip (left two subfig). Assembly of a leg (middle) showing initial, frapping and folded stages. Two hip designs tested (right).

In chassis versions prior to \#6 we used hot glue (Surebonder All purpose Glue Sticks) to adhere the foam-core plates together in locations where rigid bonds were needed. Later versions were without glue, requiring fewer materials and tools.

FABRICATION - The fiberboard of the foam-core is flexible, allowing it to be used as a flexure material itself. Such flexures can easily be 
reinforced with fiber tape to give them higher durability. However, they are limited in their direction of folding - able to fold away from the cut side but not toward it. To allow folding in both directions, we cut alignment features into the boards, allowing them to be flipped over in the laser cutter and cut accurately from both sides ("T" shaped slots in Fig. 3).

Our procedure was: (1) Cut anchoring slots and anchor foam-core on alignment fixture; (2) Affix alignment fixture in laser cutter; (3) Cut first side; (4) Flip plate and lock on alignment fixture; (5) Cut second side; (6) Release part, tape and fold.

The pattern of cuts to make a hip is shown in Fig. 3. Cuts made on both sides create the outline of the hip piece, whereas cuts made on only one side create bending joints.

DESIGN - Fig. 2 lists the many design iterations we went through, starting with scaled versions of RoACH. ${ }^{10}$ In all revisions the hip was driven by a crankshaft through hinged plates and a parallelogram linkage that converted the circular motor motion into a stepping motion. Our \#1 chassis (Fig. 1) was designed by an undergraduate senior design team based on RoACH, and the body-long drive plates on each side of the robot twisted and bent compromising to robot's ability to move. Kinematic singularities and low foot clearance also interfered with smooth operation. Simply scaling up the Berkeley designs failed. We believe this is because structural properties like plate stiffness do not scale geometricaly. The increase in mass-specific compliance with a $\times 10$ increase in size made the drive-train design unusable.

For \#2 we widened the flexures to resist torsional loads perpendicular to the hinge axis, and added stiffener ribs to many of the plates. This design (Fig. 1) was also unable to bear its own weight, but it had more favorable leg clearance in dry-dock.

In the \#3 chassis (Fig. 1) we eliminated the robot-spanning drive plates, opting for six motors driving each hip independently. This choice represents a scaling effect: with increasing size, it becomes more favorable to add motors than to add robot-spanning drive transmissions.

The design of the legs for revisions 3 and 4 of the chassis (\#3 in Fig. 1) is shown in Fig. 3, with mechanism illustrated in Fig. 4. While reliable at slow speeds on flat ground, one of the drive train parallelogram linkages occasionally flipped and jammed in an anti-parallelogram configuration when the leg encountered high loads. An additional prism on the top plate of the drive-train parallelogram linkage resolved this issue by preventing it from buckling, allowing chassis 5 to be reliable at over 40 RPM (\#4 in sFig. 1). 


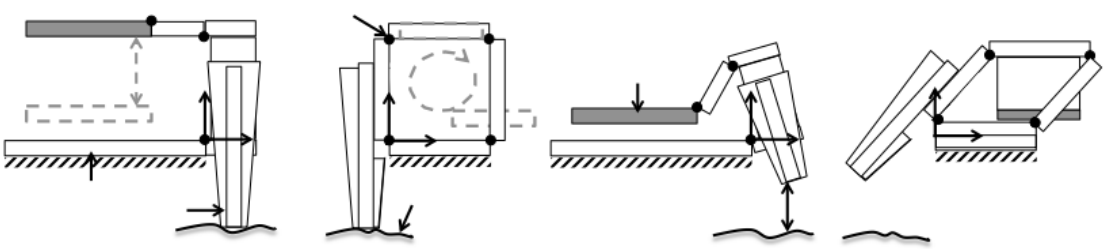

Fig. 4. The hip mechanism depicted in the stance (left pair) and swing (right pair) positions, as seen from the front and side. Motion of the crankshaft moves the link (gray filled box) in a circle (dashed arrows)

In the alternating tripod gait we used middle legs bear larger loads. We reinforced the middle hips on each side of the robot against off-axis twisting by adding an additional four-bar linkage attached to the hinge above the leg (Fig. 3 rightmost). We validated this addition by measuring structural stiffness of each leg design along the vertical axis, as expressed by a Hooke's-law spring constant, by manually moving the motor to equally spaced angular "steps" in its cycle and using a force gauge on each leg to judge the amount of force required to displace the foot, with displacements measured by a ruler to $\pm 1(\mathrm{~mm})$ (see Fig. 5). During the stance phase (i.e. when the leg is vertical and supporting weight) the reinforced legs were significantly stiffer and able to support weight. Insights gained from this reinforcing structure may play a role in future designs, even though chasis 5 and up do not use it.
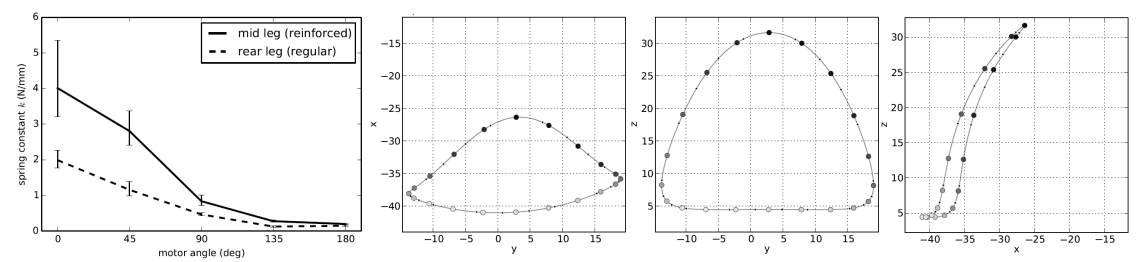

Fig. 5. Stiffness of legs to weight-bearing loads by motor phase for the two designs $\left(n=1\right.$; left panel). Note higher stiffness in stance $\left(0^{\circ}\right)$ than swing $\left(180^{\circ}\right)$, as desired.The reinforced flexure has a higher stiffness and can support a larger load.Error bars based on displacement measurement uncertainty. Kinematic model of chassis \#4 toe (orthographic views; corresponding points colored indentically in all projections; $z$ vertical; $y$ in direction of motion).

KINEMATICS - To move forward and back we produced an "alternating tripod gait" - front, middle and hind legs on alternating sides moving 
in sync. This gait is used by most hexapedal animals at moderate to high speeds within their speed range. ${ }^{11}$ Motion of each foot is theoretically determined by the 1-DOF kinematic constraints of its linkage, which is similar to that of. ${ }^{10}$ Under no load (dry-dock) conditions this is the trajectory graphed of Fig. 5 (with accuracy of $\pm 1 \mathrm{~cm}$ from possible errors in linkage dimension measurements). Leg moves vertically in the saggital plane around mid-stance, then rapidly swings outward at the end of stance to recycle forward. We designed the drive train linkage to give a $>60^{\circ}$ angle in mid-swing, for foot clearance of half a leg length.

SOFTWARE \& CONTROL - The control software for our robot used the pyckbot library developed for the 2010 ICRA Planetary Challenge. ${ }^{12}$ pyckbot comprises: (1) the logical library which identifies a variety of module architectures and busses, and represents the modules as a collection of python Module objects; and (2) the joy framework, which provides a pygame based event loop used to read user commands from keyboard, mouse, game controllers and mini slider boards, and a cooperative multithreading based collection of behavior building-blocks.

When configured in free rotation mode, the Dynamixel MX64 motors we used accept desired speed commands. Built in position commands did not allow full rotation of the motors. Instead, each motor was wrapped by a state-estimator and proportional feedback controller object, which proved sufficient to keep the motors in sync with centrally commanded reference angles and each other.

COST - The creation of the robot's chassis 4 required two $76.2 \mathrm{~cm} \times$ $101.6 \mathrm{~cm}$ pieces of $0.5 \mathrm{~cm}$ thick foam-core boards, one $76.2 \mathrm{~cm} \times 50.8 \mathrm{~cm}$ piece of $1.3 \mathrm{cmthick}$ foam-core board, $150 \pm 25 \mathrm{~cm}$ of cross-fiber reinforced tape and $280 \pm 50 \mathrm{~cm}$ of length-wise reinforced fiber tape. At the current market prices (amazon.com), this is a total of 18.01 USD in foam-core and $1.26 \pm 0.21$ USD in tape - less than 20 USD altogether. The focused costs, i.e. the costs associated with the modules, were approximately 300 USD per leg. Thus, through focused modularity, over $98 \%$ of the materials cost is preserved from one design iteration to the next.

Each hip/leg mechanism took approximately $10 \mathrm{~min}$ to laser-cut and $10 \mathrm{~min}$ to assemble (times rounded to nearest $5 \mathrm{~min}$ ). The two middle hips required an extra $10 \mathrm{~min}$ each to manufacture and attach the reinforcing four-bar linkages. Each hip/leg assembly required $5 \mathrm{~min}$ to attach to the foam base plate. The base plate needed mounting holes and slots cut to accommodate the frapping tape, which required 10 min to prepare. Each motor was attached to the base and linked to the hips in 5 min. This gave an 
overall build time of approximately $3.5 \mathrm{hr}$ for the plate-and-flexure mechanisms, the designs of which we hope to iterate.

SPEED - We measured walking speeds of chassis 4 as a function of crank-shaft rotation speed in RPM, by using a videocamera calibrated to an object of known size. Results at RPMs 15, 25 and 32 were 15, 21, and 30 $\pm 3(\mathrm{~cm} / \mathrm{s})$. At higher speeds, the motors lost coordination and the gait was less effective - presumably because of higher loads. Chassis 5 was able to walk at a nominal $42 \mathrm{RPM}$ without losing gait coordination; this difference is being further investigated.

CONCLUSION - In this work we demonstrated a manufacturing process capable of fabricating a functioning ambulatory robot chassis and mechanisms at the meter scale, allowing them to easily interact with humanscaled environments.

The focus here has been to develop the underlying manufacturing process and show that it facilitates rapid iteration. Future work will provide experimental validation of robustness and performance characteristics of robots produced by this process. The aim will be to achieve design-test iterations of robot mechanisms, conducting actual experiments in difficult to model real-world environments, at rates comparable to those possible in software development. Such rapid design iteration could lead to dramatic performance improvements, while still maintaining low cost and short robot production time.

Our robot mechanisms come from a rich family of designs previously explored by SCM, suggesting many other robots can be produced with our paradigm. Future work will enrich the library of robot designs and improve fabrication rate and quality. Complexity and cost of the robot are focused in the actuation and computation modules, with over $98 \%$ of the cost being a one-time investment in the modules. Since the robot can be built rapidly, using only a laser cutter and a few inexpensive materials, we have shown that focused modularity indeed allows a robot to be fabricated from a library of designs quickly and at low cost. This suggests building robots on-the-fly when their functionality is required.

ACKNOWLEDGMENT - Supported in part by Army Research Office grant W911NF1210284 to S. Revzen. We thank J. Brown, S. Desousa, E. Eklov, D. Litz, A. Myers, D. Zhao, C. Schaffer and E. Revzen. 


\section{References}

1. J. C. Larsen, Locomotion through morphosis: development of the modular robotic toolkit - locokit, Master's thesis, U. South Denmark (2011).

2. M. Yim, P. J. White, M. Park and J. Sastra, Modular self-reconfigurable robots, in Encycl. of Complexity and Systems Science, 2009 pp. 5618-5631.

3. A. M. Hoover, E. Steltz and R. S. Fearing, Roach: An autonomous $2.4 \mathrm{~g}$ crawling hexapod robot, in IEEE Int. Conf. on Intelligent Robots and Systems, (Nice, France, 2008).

4. P. Birkmeyer, K. Peterson and R. S. Fearing, Dash: A dynamic $16 \mathrm{~g}$ hexapedal robot, in Intelligent Robots and Systems (IROS). IEEE/RSJ International Conference on, 2009.

5. K. Y. Ma, P. Chirarattananon, S. B. Fuller and R. Wood, Science 340, 603 (2013).

6. K. L. Hoffman and R. J. Wood, Autonomous Robots 31, 103 (2011).

7. J. Whitney, P. Sreetharan, K. Ma and R. Wood, Journal of Micromechanics and Microengineering 21 (2011).

8. A. M. Hoover and R. S. Fearing, Fast scale prototyping for folded millirobots, in IEEE Int. Conf. on Intelligent Robotics and Automation, 2008.

9. R. C. Hibbeler, Statics and mechanics of materials (Prentice Hall, 2011).

10. D. W. Haldane, K. C. Peterson, F. L. G. Bermundez and R. S. Fearing, IEEE Int. Conf. on Robotics and Automation, 3279 (may 2013).

11. P. Holmes, R. J. Full, D. E. Koditschek and J. M. Guckenheimer, SIAM Review 48, 207(June 2006).

12. S. Revzen, J. Sastra, N. Eckenstein and Yim, Ckbot platform for the icra 2010 planetary challenge, in Workshop "Modular Robots: The State of the Art", Proceedings of IEEE ICRA conference, 2010. 